\title{
Métodos cuantitativos para el análisis de políticas públicas
}

\author{
Cardozo Brum, Myriam*
}

\section{Resumen}

El artículo se propone analizar los aportes y limitaciones de los métodos cuantitativos en cada etapa del proceso de políticas públicas. Para ello se realiza una revisión documenta que comienza con la definición del campo multidisciplinario que estudia estas politicas, revisando sus antecedentes, enfoques y fases de análisis. A continuación se exponen y analizan críticamente los diferentes métodos cuantitativos que se emplean en cada una de las últimas. También se realiza una síntesis de los principales asuntos discutidos respecto del tema en cuestión, en recientes años, evaluando las perspectivas futuras de estas aportaciones. Se concluye que los métodos cuantitativos constituyen una valiosa aportación, no siempre explorada, al Análisis de Politicas Públicas, slempre y cuando se tenga presente el conjunto de supuestos en que se fundamenta cada método, por lo tanto, sus limitaciones, y que éstos se compiementen con estudios cualitativos que permitan una adecuada integración de las racionalidades técnica y politica.

Palabras clave: Métodos cuantitativos, políticas públicas, proceso de enseñanza.

\section{Quantitative Methods for the Analysis of Public Policy}

\begin{abstract}
This article analyses the advances and limitations of quantitative methods in each stage of the process of public policy analysis. In order to do this, a documentary revision was made beginning with the definition the multidisciplinary field that studies these policies, reviewing their antecedents, focuses and analytical phases. This is followed by an exposition and critical analysis of the quantitative methods used in each of the above fields. The principal themes discussed with respect to this topic in recent years are synthesized, evaluating the future perspectives of each contribution. The conclusion is that quantitative methods are a valuable support, and one that is not always taken into account in public policy analysis, but only when the set of suppositions that underlie each method, as
\end{abstract}

Recibido: 00-05-24. Aceptado: 01-02-05

* Coordinadora de la Licenciatura en Politica y Gestión Social, U.A.M (X) México, D.F. Email: mcardozo@cueyatl.uam.mx, myriamcardozo@ hotmail.com 
well as its limitations are understood. These should be complemented with qualitative studies that allow for an adequate integration of technical and political rationalities.

Key words: Quantitative methods, public policy, teaching process.

\section{Aspectos conceptuales}

Cuando se realiza una rápida revisión de definiciones $y$ enfoques en el tema, se encuentra un enorme abanico de alternativas, que no es el momento de revisar exhaustivamente ${ }^{1}$.

Esquemáticamente, podemos decir que hay un importante grupo de conceptos de políticas públicas que las consideran como los lineamientos generales que delimitan el conjunto de alternativas a considerar en decisiones y acciones más concretas para el logro de objetivos, en el marco de un plan. Se ubican fundamentalmente en el momento de la formulación de políticas, resaltan como sus principales elementos a las previsiones y decisiones y enfatizan el uso de modelos de análisis de tipo racional-técnico.

Por otro lado, hay definiciones que las conciben como el conjunto de acciones y omisiones relativas a una cuestión que hay que analizar y evaluar a partir de la utilización de modelos de carácter explicativo como el burocrático-organizacional o el político.

Pretendiendo hacer una síntesis selectiva de las anteriores propongo definir nuestro objeto de estudio como el resultado de un proceso de sucesivas tomas de posición, que se concretan en un conjunto de decisiones, acciones u omisiones, asu- midas fundamentalmente por los órganos del Estado, que traducen, en un lugar y período determinado, la respuesta preponderante del mismo frente a los problemas de la sociedad (Cardozo, 1994).

En relación a esta propuesta hay que resaltar algunas cuestiones:

- Hablamos de un proceso de sucesivas tomas de posición, por lo que descartamos que una decisión aislada, especifica, constituya una política.

- Identificamos como actor relevante en la elaboración de políticas al Estado. Esto no significa que neguemos importancia a las organizaciones civiles, movimientos sociales, o ciudadanos en general, sino que pensamos que, a pesar del auge de las denominadas organizaciones no gubernamentales (ONG's) que actúan en asuntos de políticas públicas, su papel ha sido fundamentalmente el de oficiar como grupos de presión para que el Estado asuma posición frente a un problema o recoja una propuesta, no pudiendo resolver por sí mismas más que los asuntos de pequeña escala, debido a que no es su objetivo reemplazar al Estado en su responsabilidad ni los recursos que disponen les permitirían esta sustitución. Es asi que el Estado continúa siendo

1 Consultar al respecto: Cardozo Brum, Myriam, "Análisis de politicas de salud, "Antecedentes y enfoques de políticas públicas en América Latina". 
considerado como el principal actor, capaz de ofrecer bienes y servicios que satisfagan las necesidades sociales. Además, en el contexto de los gobiemos democráticos, la participación social en los procesos de políticas públicas se vuelve cada día más relevante.

- El Estado y, más específicamente, los gobiernos, no actúan de manera absolutamente coordinada, racional y consistente, por lo que podemos encontrar instituciones al interior de los mismos con posiciones diferentes; por esta razón, sólo podemos identificar tendencias predominantes. Estas pueden ser asumidas incluso por omisión, pues no hacer nada frente a un problema también implica adoptar una posición.

- El proceso al que hacemos referencia se puede desagregar en un número variable de fases. Una posibilidad es la siguiente:

a. Un primer momento que denominamos de gestación, en el que surge un problema, demanda o simplemente necesidad en la sociedad. La misma puede ser planteada por los grupos sociales afectados, por sus representantes, por organizaciones intraburocráticas o incluso por organismos internacionales. Esta fase concluye cuando la institución gubernamental responsable del tema en cuestión, lo incorpora en su agenda de asuntos a resolver.

b. La segunda fase incluye un diagnóstico y definición del problema por parte del organismo estatal, lo que condicionará el conjunto de alternativas de solución que comenzará a generar y evaluar "a priori" para seleccionar la que considere más apropiada. El diseño detallado de ésta cerrará la etapa de formulación.

c. A continuación será necesario traducir en acciones concretas la política antes decidida, de forma de impactar el problemas social que le dio origen. Se trata de la fase de implementación.

d. Transcurrido el tiempo necesario para poder medir los resultados e impactos alcanzados, podrá procederse a realizar una evaluación de la política para poder describir el proceso que se llevó a cabo, explicar sus determinantes y condicionantes y calificar su grado de efectividad.

La evaluación permitirá decidir si la política continúa aplicándose y si lo hará con o sin modificaciones, pudiendo dar lugar a la repetición de todas o algunas de las fases señaladas. Lógicamente, el proceso descrito no siempre se presenta en la práctica con la linealidad con la que aquí analíticamente se planteó.

La participación social se presenta fundamentalmente en los extremos del proceso descrito, pero eso no significa que desaparezca totalmente en las dos fases intermedias. Esto nos induce a realizar a continuación una breve consideración en relación a la forma en que se han venido modificando los límites entre lo público y lo privado.

En México, como en la mayoría de los países latinoamericanos, la aplicación del enfoque neoliberal, ha movido las antiguas fronteras entre lo público y lo privado, ya que la sociedad organizada en ONG's, empresas privadas, asociaciones, etc., ha asumido algunas funciones que antes desarrollaba el Estado. Mien- 
Métodos cuantitativos para el análisis de politicas públicas

Cardozo Brum, Myriam

tras continúa considerándose necesaria la existencia de una política gubernamental de relaciones exteriores o de defensa y seguridad pública, en materia económica y social (empleo, distribución del ingreso, educación, salud, vivienda, etc.) se aprecian importantes retrocesos.

El contenido descrito hasta aquí como políticas públicas, es estudiado por un conjunto amplio de disciplinas que se integran en lo que podemos denominar Análisis de Políticas Públicas y, que en los E.U.A. puede aparecer bajo un gran número de rótulos (Public Policy, Public Decision, Political Sciences, Policy Analysis, Policy Evaluation, Programs Evaluation, etc.). Este campo multidisciplinario recoge asi tanto los aportes de la política, la sociologia, la antropologia, la psicología, la administración, la economía, como los de la estadística o las matemáticas. Todas ellas confluyen a la búsqueda de soluciones a las problemas (fundamentalmente a través del enfoque normativo, con un fuerte contenido de análisis cuantitativos) y a la descripción y explicación ex-post de la política implementada (con énfasis en estudios cualitativos). En todo momento deben combinar los análisis de factibilidad técnica con los de viabilidad política; el incremento de la eficiencia social y el de la equidad distributiva.

\section{Antecedentes}

Al igual que la mayoría de los enfoques cuantitativos aplicados al estudio de la administración (modelos para toma de decisiones, investigación de operaciones, etc.), el análisis de las políticas públicas se desarrolla después de la Segunda Guerra Mundial, fundamentalmente en los E.U.A. Dicho análisis se realiza con la intención de mejorar la eficiencia de los procesos vinculados a la toma de decisiones Bachus y Webster (1981); Stockey y Zeckhauser (1975); Quade (1975).

En el mismo contexto, surgen nuevos planteos cuestionando la pertinencia de los enfoques cuantitativos en este campo Lindblom (1989), Dror (1970), Grindle (1977), Braybrooke y Lindblom (1963), si bien estos continúan constituyendo el enfoque predominante en los E.U.A.

En la década de los setentas, a nivel latinoamericano, se retoma el cuestionamiento anterior, poniendo el énfasis en la descripción y explicación de las acciones y omisiones del Estado en materia de políticas O'Donnell y Oszlak (1976), Oszlak (1980), Testa, s/n Unikel (1976); y tratando de adaptar el análisis al tipo de estado y régimen políticos vigentes en diferentes momentos y paises del área.

Es importante señalar también el esfuerzo realizado por autores como Allison (1971) y Steinbruner (1974) que, desde el enfoque de decisiones, intentar aplicar separadamente los dos planteos anteriores a un mismo problema. Resta por profundizarse la discusión sobre la posibilidad de complementación entre ellos.

Tampoco los europeos han quedado al margen de este esfuerzo, particularmente en Francia Nioche (1982) Mény y Thoenig (1992); Crozier (1987); Sfez (1990) Leca y otros (1985) Quermone y Rouban (1983), donde si bien el enfoque cuantitativo tiene fuerza, es al explicativo al que se le presta mayor atención.

En la actualidad, otros países europeos como Gran Bretaña, Alemania, Países Bajos, Suiza, Noruega, etc. han reali- 
zado importantes avances, fundamentalmente en materia de evaluación. Otro tanto sucede con el caso de Canadá en América.

Dentro del contexto latinoamericano, México comenzó su incursión en el tema en la década de los ochenta con algunas investigaciones e introducción de programas de Análisis de políticas Públicas en algunos planes de estudio de postgrado en Administración Pública. En la actualidad existen programas de maestría, especialidad y múltiples diplomados, específicos en el tema.

\section{Papel de los métodos cuantitativos}

A continuación analizaremos las técnicas cuantitativas que se emplean en las diferentes etapas del proceso antes descrito.

\subsection{En la gestación de problemas}

La principal función del análisis cuantitativo en esta fase es la de relevar información estadística que permita describir la situación (diagnóstico) y prever su posible tendencia a futuro (pronóstico). Se utilizan cálculos de tasas, medidas simplificadoras de tendencia central (medias, medianas y modas), series de tiempo, extrapolaciones, etc. Es recomendable acompañar las segundas con medidas de dispersión (desviación standard, varianza), para profundizar en el conocimiento de la diversidad que aquellas tienden a "suavizar".
En las últimas décadas, las proyecciones ceteris paribus han venido a enriquecerse con los estudios de prospectiva basados en el diseño de escenarios futuros, ante la eventualidad de que las condiciones, antes consideradas constantes, pudieran modificarse por la presentación de eventos que generalmente no se prevén. El conocimiento de los escenarios alternativos futuros es aprovechado como oportunidad para incidir, en la medida de lo posible, mediante la implementación de estrategias que puedan modificar el curso actual de las tendencias de las variables sustantivas del problema en estudio, facilitando su mejor evolución. En este caso se resalta la importancia de la incertidumbre como la característica más relevante de los problemas que ameritan la toma de posición estatal. Por ello conjugan en su tratamiento la utilización de modelos matemáticos como la simulación y el análisis estadistico de encuestas, junto con la recolección de opiniones más cualitativas como es el caso de la consulta a expertos mediante la técnica DELPHI (Sach, 1980).

En esta etapa, considero que el análisis social y político resulta relevante y que los métodos cuantitativos actúan sólo como elemento de apoyo.

\subsection{En la formulación de políticas públicas}

La formulación corresponde a la segunda fase del proceso, pero es la primera en que el Estado juega un papel trascendente, ya que, aunque parta de una propuesta de la sociedad, es a través de sus instancias técnicas, políticas y administra- 
tivas en que se definirán, en la enorme mayoría de los casos, los objetivos a perseguir, se analizarán las alternativas disponibles, se evaluarán sus consecuencias, se discutirán internamente las opiniones divergentes, se recibirán las presiones de los grupos sociales, se negociará con ellos, etc., para llegar finalmente a diseñar la política que en esa área específica se piensa aplicar en un lugar y periodo determinado (Cardozo, 1983).

Tal vez por la razón anterior, y por tratarse de decisiones públicas concretas, es que se ha desarrollado una gran diversidad de métodos cuantitativos que sirven de apoyo a esta fase, sobretodo en el nivel de programas y proyectos especificos. Su utilización se enmarca dentro del concepto de racionalidad y eficiencia redefinidos para la gestión pública, pero propios de la adecuación de "medios a fines", aunque estos últimos resulten múltiples y, a menudo, contradictorios entre si. Sin embargo será requisito indispensable de la aplicación de técnicas cuantitativas, el tener objetivos claramente definidos y jerarquizados. Su utilidad dependerá también de que los niveles previos de la formulación hayan sido adecuadamente cubiertos (determinación de los objetivos sociales, las megapolíticas y las politicas sustantivas).

La mayoría de los métodos cuantitativos disponibles para esta etapa son de carácter optimizador, pretendiendo elegir el mejor proyecto con base en algún criterio definido, como es el caso del análisis financiero, costo-beneficio y costo-eflcacia o la mejor combinación de los mismos cuando se aplica programación IIneal. Sin embargo, también se dispone de métodos participativos que sólo buscan hacer la elección entre los proyectos que satisfagan más a quienes resultan afectados por los mismos. Es el caso de los análisis multicriterios como el denominado ELECTRA (Cardozo, 1993).

Los métodos anteriores se desarrollan en condiciones de certidumbre, como si los datos que se manejan fueran absolutamente ciertos, seguros, y a lo sumo, se realizan análisis de sensibilidad. También pueden utilizarse árboles de decisión para decidir en condiciones de riesgo, cuando es posible atribuir probabilidades a los posibles eventos futuros y teoria de juegos para los casos en que la incertidumbre es relevante.

Otra técnica de medición cuantitativa, el análisis de factibilidad (F.A.T.) se ha creado con características absolutamente diferentes a las anteriores, pues su objetivo es fundamentalmente político : tratar de anticipar la posible respuesta de los grupos de interés ante la formulación de una política nueva o la reformulación de la vigente.

Finalmente, existen también una serie de técnicas cuantitativas que no constituyen en si métodos para tomar decisiones pero que pueden servir como herramientas de apoyo para alimentar los modelos anteriores. Se trata de las ecuaciones en diferencias, la teoria de colas o líneas de espera, los modelos de simulación y las cadenas de Markov (Cardozo, 1993).

Esta es la fase en la que se han aplicado más técnicas cuantitativas y donde han sido más útiles sus aportaciones en términos de introducción de orden, eliminación de contradicciones, estructuración de los problemas, sistematización de la in- 
formación, etc. Sin embargo, si no se conocen los supuestos y limitaciones de cada método, se puede correr el riesgo de adoptar decisiones erróneas.

\subsection{Durante la implementación de acciones}

Las políticas públicas son llevadas a la práctica a través de acciones y omisiones, basadas en una toma de decisiones de carácter táctico-operativo, a diferencia de la etapa de la formulación en que las decisiones ameritaban ser calificadas de estratégicas.

El análisis cuantitativo puede aportar en esta fase sólo algunas técnicas de control de gestión que miden la eficiencia y eficacia con que está siendo implementada la política formulada, como los denominados tableros de control o cuadros de mando, que controlan las variaciones de sus indicadores de factores-clave, dentro de los niveles estándares predeterminados (Medina, 1993).

También puede aplicarse el método de ruta crítica (PERT-CPM) de investigación de operaciones, en que se calendariza un proyecto buscando minimizar su duración y/o costos. Como todos los métodos de optimización, su aporte depende de la precisión con que puedan determinarse los datos que le son necesarios.

\subsection{En la evaluación de resultados}

Algunos métodos cuantitativos citados para hacer evaluación de proyectos (análisis costo-eficacia, costo-beneficio, ELECTRA) pueden adaptarse tam- bién para evaluar resultados, sustituyendo lo que antes eran previsiones por los datos históricos realmente obtenidos. Lógicamente, en los métodos optimizadores, ese criterio se pierde porque generalmente las posibles alternativas de un proyecto no se ponen en práctica y por lo tanto, se carece de posibilidades aceptables de comparación.

La mayor parte de las evaluaciones cuantitativas de resultados consisten en mecanismos de medición de simples porcentajes de eficacia alcanzada, con base en la meta programada.

Las técnicas estadísticas, por su parte, permiten dar tratamiento cuantitativo a la aplicación de encuestas de opinión, otro de los mecanismos de evaluación de resultados más empleado.

Algunas técnicas más especificas que intentan hacer una medición más completa del impacto alcanzado serían los análisis de excedentes de productividad, los balances sociales y sobre todo los estudios quasiexperimentales ${ }^{2}$.

Los primeros, como su nombre lo indica, miden la evolución de la productividad en una organización, la forma en que participan en su generación los distintos factores de la producción y la distribución del excedente generado. Permiten clarificar las transferencias que el sector público hace al privado por medio del sistema de precios y tarifas y planear el excedente que se desea generar y la forma como se le quisiera distribuir, no sólo a nivel de empresas individuales sino en forma agregada para toda una rama o sector.

2 Consultar para una revisión de estos métodos: Cardozo Brum, Myriam, "La función de la evaluación en las organizaciones no lucrativas". 
Los balances sociales tienen por objetivo complementar por medio de un conjunto de indicadores cuantitativos y explicaciones cualitativas, la información presentada por el baiance económico-financiero tradicional en relación a los esfuerzos alcanzados y los logros obtenidos en el campo social.

Los estudios quasiexperimentales se utilizan para establecer relaciones de causalidad: medir el impacto de programas mediante su aplicación a una población (caso) que es comparada con otra similar en la que el programa no se pone en práctica (control). Si la condición de similitud previa de los grupos es bastante grande, lo que es muy difícil de lograr en ciencias sociales, es probable que las diferencias finales halladas se deban al programa. Sin embargo, es necesario deslindar, y muy difficil de realizar, que las diferencias puedan haber sido causadas por variables no controladas, fundamentalmente de tipo contextual.

Como puede verse la medición cuantitativa de resultados e impactos presenta menos nivel de desarrollo de lo que sería deseable en nuestro interés de identificar la efectividad de las políticas y tomar decisiones sobre su finalización o continuación, con o sin modificaciones.

\section{Situación actual y perspectivas de su desarrollo aplicado}

En una revisión hemerográfica recientemente realizada (Cardozo,1999) sólo se encontró un estudio ex-ante, propio de la etapa de formulación. Se trata del trabajo de R. Hahn (1995); de análisis de costo-eficacia para la elección de gasolinas y tecnologías de los automóviles para el control de los efectos contaminantes, lo que podría estar indicando que, al menos en los países desarrollados ya no resultan originales e interesantes para su publicación.

En cambio siguen siendo de interés los modelos de simulación que permiten predecir tendencias y probar el impacto de diferentes alternativas de políticas, como es el caso del estudio de Correa (1994); en relación con las alternativas disponibles para controlar la epidemia del SIDA.

Lo mismo sucede con los modelos de teorías de colas, que se ejemplifican en un trabajo de Peterson, et al (1995); para resolver el problema de la congestión de los aeropuertos o la prospectiva de escenarios múltiples, aplicada por $B$. Barraqué (s/f) al combate contra el ruido.

En cuanto a la evaluación ex-post, en la misma revisión se encontró la utilización por parte de Borges, et al (1994); de un modelo matemático no paramétrico para la medición de la eficiencia productiva y la explicación de las diferencias en el nivel municipal.

Por su parte, Figlio (1995); aplica series de tiempo para medir el impacto de un programa que elevó la edad en que se autoriza a beber públicamente alcohol en los EUA.

Si comparamos el papel jugado por los métodos cuantitativos en estas evaluaciones, que corresponden a las dos fases del proceso de políticas públicas en que resultan más útiles, con los estudios de carácter cualitativo que se publican sobre el 
tema, considero que esta última perspectiva está siendo más productiva y probablemente, por diferentes razones, más aceptada por los lectores. La tendencia muestra más interés en el desarrollo de los segundos, que se supone predominarán en los próximos años, salvo que se logre mayor realismo en el desarrollo de modelos matemáticos aplicados, lo que implica, entre otros aspectos, poner el énfasis en modelos de satisfacción en lugar de los optimizadores, debido a que ellos parecen captar mejor la complejidad de las situaciones sociales y políticas.

\section{Conclusiones}

En el análisis de las políticas públicas, los métodos cuantitativos no son siempre usados con la neutralidad que aqui han sido expuestos, sino que a menudo son manipulados para justificar y legitimar decisiones adoptadas con base en otros intereses, en cuyo caso el made10 incrementalista de Lindblom explica mejor estos procesos.

Ellos constituyen una aportación valiosa al Análisis de Políticas Públicas, siempre y cuando se tenga presente el conjunto de supuestos en que se fundamentan, y por lo tanto, las limitaciones que presentan, y que se les complemente con el estudio de los aspectos cualitativos, siempre importantes en este campo, como son los problemas de negociación, consenso, legitimidad, etc. De esta manera se obtiene la adecuada integración de las racionalidades técnica y política, necesarias en nuestro campo.

Urge el desarrollo de modelos con mayor capacidad para captar situaciones complejas en su dinámica de cambio que superen las enormes simplificaciones de los enfoques optimizadores de tipo normativo.

\section{Bibliografía citada}

Allison, Graham (1971). Essence of Decision, Little, Brown and Co, E.U.A.

Bachus y Webster (1981). " Politica burocrática; un paradigma y algunas implicaciones de políticas", Cuadernos de Estados Unidos, No. 10, México, CIDE A.C.

Braybrooke y Lindblom (1963). Strategy of Decision: policy evaluation as a social process, EUA, Free Press.

Barraqué, Bernard (s/f)". Le bruit des transport. Politiques et techniques de rèduction", en Problemes Politiques et Sociaux, No. 734.

Borges K., Kerstens W., Moesen y Vanneste J. (1994). "Explaining differences in productive efficiency: an aplicacation to Belgian municipalities", en Public Choice, vol. 80.

Cardozo Brum, Myriam (1983). Formulación de politicas públicas, Material Docente No.5, Serie Administración Pública, México, CIDE A.C.

Cardozo Brum, Myriam (1992). "Análisis de Políticas de salud, ¿Un área nueva?", en Revista de Salud Pública, Vol. 31 , No.5, INSP, México, Sept. Oct. 1989 o Antecedentes y enfoques de Políticas Públicas en América Latina," ponencia presentada al Congreso de Administración Pública, Universidad de Cuyo, Mendoza, Argentina, Noviembre.

Cardozo Brum, Myriam (1993). "Evaluación de las Politicas Públicas: problemas, metodologias, aportes y limitaciones", en Revista de Administración Pública No. 84, México, INAP. 
Cardozo Brum, Myriam (1994). "Politicas Públicas: conceptos, antecedentes y campo profesional" conferencia presentada en el Programa Inaugural Internacional de la Maestría en Politicas Públicas, México, UAM-X.

Cardozo Brum, Myriam (1998). Evaluación: conceptos, metodologias y aplicaciones en la educación", en Luis Bernecos (coord. Y. Editor). La Evaluación en el sistema modular. México, UAM-X, 1998, $Y$ "La función de la evaluación en las organizaciones no lucrativas", en Revista Administración y Organizaciones No. 1, México, UAM-X.

Cardozo Brum, Myriam (1999). "Experiencias recientes de evaluación en organizaciones no lucrativas", Revista Admlnistración y Organizaciones, No. 2, México, UAM-X.

Correa, Hector (1994). "A model for the analysis of optimal policies to control the AIDS epidemic", en Journal Of Policy Modeling, Vol. 16, No. 1.

Crozier, Michel (1987). Estado modesto, Estado moderno, México, FCE.

Dror, Yehezkel (1970). "Prolegomena to policy sciences", en Policy Science, No. 1 , EUA.

Figlio, David (1995). The effect of drinking age laws and alcohol-related crashes: time - series evidence from Wiscon$\sin ^{\prime \prime}$, en Journal of Policy Analysis_and Management, Vol. 14, No. 4.

Grindle, Merilee (1977). Burocrats, politiciants and peasants in Mèxico, EUA, University of California Press.

Hahn, Robert (1995). "Choosing among fuels and technologies for cleaning up The air", en Joumal of Policy Anaysis and Management, Vol. 14, No. 4.

Leca, Jean (1985) y otros. La theorie politique, Francia.

Lindblom, Charles (1959). "The science of muddling through", en Public Administration Review, Vol. 19, EUA.
Nioche, Jean Paul (1982). De l' evaluation a I' analyse des politiques publiques, Revue Francaise de Sciences Politiques No. 1, Francia.

Medina, Alejandro y José Mejlas (1993). control en la implementación de la política pública, México, Plaza y Valdés.

Mény y Thoenig (1992). Las políticas públicas, España, Edit. Ariel.

O' Donnell y Oszlak (1976). Estado y políticas estales en América Latina: hacia una estrategia de investigación, Argentina, CENDES.

Oszlak, Oscar (1980). Políticas públicas y regimenes políticos: reflexiones a partir de algunas experiencias latinoamericanas, Argentina, CENDES.

Peterson M.; Bertsimas D.; y Odoni A. (1995). "Models and algorithms for transient queing congestion at airports", en $\mathrm{Ma}$ nagement Science, Vol 41, No. 8.

Quade, E. (1975). Analysis for public decisions, EUA, A merican Elservier.

Quermone y Rouban (1983). L'evaluation des politiques publiques et le controle politique, en Coloquio La Evaluación de las políticas Públicas, París.

Sach, Wladimir (1980). Diseño para un Futuro. Ensayo sobre métodos e importancia de la planeación prospectiva, México, Fundación Javier Barrios, Sierra.

Sfetz, Lucien (1990). Crítica de la decisión, México, FCE.

Steinbruner, John (1974). The cybernetic theory of decision, Princeton University Press, New Jersey.

Stockey y Zeckhauser (1978). A primer for policy analysis, EUA, w.w. N and Co.

Testa, Mario (s/fecha). Planificación y políticas de Salud, Venezuela, Universidad Central de Venezuela-CENDES.

Unikel, Luis (1976). Ensayo sobre politica de desarrollo regional en México, INFONAVIT, Año 1, No. 5, México. 